Pacific Journal of Mathematic 


\title{
RESTRICTIONS OF PRINCIPAL SERIES TO A REAL FORM
}

\author{
RONALD L. LIPSMAN
}

\begin{abstract}
In this paper I consider restrictions of nondegenerate principal-series representations of a complex semisimple Lie group to a noncompact real form.
\end{abstract}

The motivation for studying these restrictions comes from several different directions. First it is a problem of some interest to physicists. For example, a low-dimensional instance of my work occurs in [2]. Second the problem and methods of solution are closely related to the project [7], [8]. In fact I shall settle the main outstanding conjecture of [7] in this paper. Other reasons for treating this problem are: restrictions provide a means of realizing discrete-series representations of real semisimple Lie groups (see [3])-in this work we seek to enumerate which discrete series actually occur in which restrictions; it's already well-known (see e.g., the work of Moore [11]) that restrictions can be studied in order to obtain information on ergodicity of group actions on homogeneous spaces. Finally, there is an obvious connection between this problem and the problem of decomposing tensor products of principalseries representations of real semisimple Lie groups (compare Theorem 2.1 here and [9, Theorem 1]). I have some hope that techniques employed in this paper might eventually prove useful in studying tensor products of discrete-series representations.

Here is a brief description of the main results and of the organization of the paper. Let (S) be a complex semisimple Lie group, $G \cong(\mathbb{S}$ a real semisimple Lie group whose complexification is 8 . The nondegenerate principal series representations of $\$$ are induced from unitary characters of a Borel subgroup. So let $\mathfrak{B} \subseteq(\mathbb{S}$ be a Borel subgroup, $\chi \in \hat{\mathfrak{B}}$ a unitary character, and form $\pi(\chi)=\operatorname{Ind}_{\mathfrak{B}}^{s} \chi$. We are interested in a description of the irreducible components, and their multiplicities, of the representation $\left.\pi(\chi)\right|_{G}$. Since $\pi(\chi)$ is an induced representation, we begin our study by invoking the Subgroup Theorem. This requires an explicit knowledge of the $\mathfrak{B}: G$ double cosets in (8). But that data has been worked out already in [13]. We shall combine Wolf's results with the Subgroup Theorem to reduce our problem to the study of representations of the form $\operatorname{Ind}_{l i}^{G} \chi_{1}$, where $\chi_{1}$ is a unitary character of a maximally compact Cartan subgroup $H$ in $G$ (see Theorem 2.1). This is the content of $\S 2$. We then analyze the spectrum of $\operatorname{Ind}_{H}^{G} \chi_{1}$ by the technique of Anh reciprocity. That requires that we compute the direct integral decomposition of the 
restrictions $\left.\pi\right|_{H}$ for generic irreducible unitary representations $\pi$ of $G$. In $\S 3$ we carry that out for the various principal-series representations of $G$ (corresponding to the cuspidal parabolic subgroups of $G$ ); in $\S 4$ we treat discrete-series representations of $G$. Our results on the continuous spectrum are complete and definitivenamely, we prove: for all principal series representations $\pi$ of $G$, $\left.\pi\right|_{H}$ is quasi-equivalent to $\operatorname{Ind}_{Z_{G}}^{H} \omega_{\pi}$ where $\omega_{\pi}$ is the central character of $\pi$ (see Theorems 3.1 and 3.4). On the other hand, we only obtain partial conclusions for the discrete spectrum. We show that only finitely many holomorphic (or conjugate holomorphic) discrete-series representations occur in the spectrum of $\left.\pi(\chi)\right|_{G}$; and we give a partial result to support the conjecture that all nonholomorphic discrete-series representations (up to central character) appear in the spectrum (see Theorem 4.2 and Proposition 4.8). In $\S 5$ we compute the multiplicity for the continuous spectrum of $\left.\pi(\chi)\right|_{G}$. The result is that multiplicity is uniform and-with two exceptionsinfinite (Theorem 5.1). We also show that holomorphic discrete series have finite multiplicity; and we pose a conjecture on the multiplicity of nonholomorphic discrete series. Finally, $\S \S 6$ and 7 contain explicit examples. In $\S 6$ we illustrate all the results of the paper in the special case $G=\mathrm{SU}(2,1) \subseteq B=\mathrm{SL}(3, C)$. We then use these computations in $\S 7$ to show that the unitarized adjoint representation of $G=S U(2,1)$ is not unitarily equivalent to $\operatorname{Ind}_{z_{G}}^{G} 1$.

I conclude the introduction by establishing some notation and terminology. Since we shall rely heavily on the Subgroup Theorem (in both $\S 2$ and $\S 3$ ), I include its statement with notation designed to suit my purposes. Let $G$ be locally compact with $A, B \subseteq G$ closed subgroups. Let $\nu$ be a pseudo-image on $A \backslash G / B$ of Haar measure on $G$. $A$ and $B$ are called regularly related (resp. discretely related) if some $\nu$-co-null set in $A \backslash G / B$ is countably separated (resp. if some $\nu$-co-null set in $A \backslash G / B$ is countable).

THEOREM 1.1. (Subgroup Theorem). Let $A$ and $B$ be regularly related in $G$, and let $\pi$ be a unitary representation of $A$. Then

$$
\left.\left(\operatorname{Ind}_{A}^{G} \pi\right)\right|_{B} \cong \int_{A \backslash G / B}^{\oplus} \operatorname{Ind}_{B_{x}}^{B} \pi^{x} d \nu(x)
$$

where $B_{x}=B \cap x^{-1} A x$ and $\pi^{x}(b)=\pi\left(x b x^{-1}\right), b \in B_{x}$.

I shall write $\#(A \backslash G / B)$ for the Hilbert space dimension of $L_{2}(A \backslash$ $G / B, \nu)$. If $G$ is type $I$ and $\pi$ is a unitary representation of $G$, then the direct integral decomposition into irreducibles

$$
\pi=\int_{\omega \in \hat{G}}^{\oplus} n_{\pi}(\omega) \omega d \mu_{\pi}(\omega)
$$


is unambiguously determined-i.e., the class of $\mu_{\pi}$ is uniquely defined and the multiplicity function $n_{\pi}(\omega)$ is uniquely determined up to $\mu_{\pi}$-null sets. If $G$ is unimodular and type $I, I$ write $\hat{G}_{d}$ for the discrete spectrum of the regular representation of $G$. If $G$ acts on a manifold $X$, then by a principal orbit type I mean an open dense submanifold $O \cong X$ such that $G_{x}=\{g \in G: g \cdot x=x\}$ is conjugate to $G_{y}, \forall x, y \in \mathcal{O}$. The groups $G_{x}, x \in \mathcal{O}$ are called principal stability subgroups. If $A \subseteq B \cong G$ is a sequence of closed subgroups, I write $N_{B}(A)$ (resp. $Z_{B}(A)$ ) to denote the normalizer (resp. centralizer) of $A$ in $B$. Also $G^{0}$ denotes the neutral component of $G$, and $Z_{G}=$ $Z_{G}(G)$ is its center. Finally the Lie algebra of a group which is denoted by an upper case Roman letter will always be indicated by the corresponding lower case German letter.

It is my pleasure to acknowledge valuable assistance in the preparation of this paper from Nolan Wallach and Joseph Wolf.

2. Application of Wolf's theorem. Suppose 83 is a connected complex semisimple Lie group with Lie algebra $g$. Let $G \leqq \mathbb{B}$ be a real form, i.e., the connected Lie subgroup of (\$) corresponding to a real form $\mathfrak{g} \subseteq \boldsymbol{g}$. We shall assume throughout this paper that $G$ is simple and noncompact. We are interested in decomposing restrictions to $G$ of nondegenerate principal-series representations of (S). In this section, we effect a first reduction of the problem by employing the Subgroup Theorem and Wolf's results [13].

Let $\mathfrak{B}$ be a Borel subgroup of $\mathbb{B}$ with $\chi \in \hat{\mathfrak{B}}$ a unitary character. We set $\pi(\chi)=\operatorname{Ind}_{\mathfrak{B}}^{\mathfrak{s} \chi}$. The collection $\{\pi(\chi): \chi \in \hat{\mathfrak{B}}\}$ constitutes (with some duplication) the nondegenerate principal series of irreducible unitary representations of $\&$. We want to decompose $\left.\pi(\chi)\right|_{G}$. But

$$
\left.\pi(\chi)\right|_{G}=\left.\left(\operatorname{Ind}_{\mathfrak{B}}^{\circledast} \chi\right)\right|_{G}
$$

is a perfect candidate for the Mackey Subgroup Theorem. To apply Theorem 1.1 we must investigate the double coset space $\mathfrak{B} \mid \mathbb{S} / G$. Fortunately, this has been done already by Wolf [13].

THEOREM 2.1. There exist: (i) a Cartan subgroup $\mathfrak{F}$ of (S) such that $H=\mathfrak{S} \cap G$ is maximally compact among the Cartan subgroups of $G$; and (ii) finitely many unitary characters $\chi_{i} \in \hat{H}$ such that

$$
\left.\pi(\chi)\right|_{G}=\sum_{i}^{\oplus} \operatorname{Ind}_{H}^{G} \chi_{i}
$$

If $\mathfrak{S} \cap G$ is actually compact (in which case we write $T=\mathfrak{S} \cap G$ ), then there exists an element $\sigma \in \mathbb{S}$ such that $\sigma \mathfrak{S} \sigma^{-1} \subseteq \mathfrak{B}$ and 


$$
\left.\pi(\chi)\right|_{G}=\sum_{s \in W_{\Theta} \mid W_{G}}^{\oplus} \operatorname{Ind}_{T}^{G} \chi^{o s}
$$

where $W_{\Theta}=N_{\Theta}(\mathfrak{S}) / \mathfrak{S}$ and $W_{G}=N_{G}(T) / T$.

Proof. Our first observation is that $\mathfrak{B} \mid(\mathbb{S} / G=(\mathfrak{B} \mid(\mathbb{S}) / G$-i.e., there is a natural 1-1 measurable-set preserving correspondence between the double coset space $\mathfrak{B} \backslash \mathbb{S} / G$ and the set of orbits for the right action of $G$ on $\mathfrak{B} \mid \mathbb{S}$. But Wolf has proven [13] that there are a finite positive number, say $n$, of open $G$-orbits on $\mathfrak{B} \mid(S)$, and that the complement of their union is a manifold of lower dimension. This shows in particular that $\mathfrak{B}$ and $G$ are regularly related (even discretely related). Hence Theorem 1.1 applies. Let $g_{1}, \cdots, g_{n} \in \mathbb{S}$ be any collection of points with exactly one from each open double coset. Then

$$
\left.\pi(\chi)\right|_{G}=\sum_{i=1}^{\oplus} \operatorname{Ind}_{\mathfrak{B}_{i}}^{G} \chi^{g_{i}} .
$$

Note that if $x_{i}=\mathfrak{B} g_{i} \in \mathfrak{B} \mid \mathbb{S}$, then the group $\mathfrak{B}_{g_{i}}=G \cap g_{i}^{-1} \mathfrak{B} g_{i}$ is exactly the stability group of $x_{i}$ under the right action of $G$ on $\mathfrak{B} \backslash \mathbb{S}$. We now apply the results of $[13, \S \S 4-6]$. They guarantee the existence of Cartan subgroups $\mathfrak{F}_{i}, 1 \leqq i \leqq n$, such that each $H_{i}=$ $\mathfrak{F}_{i} \cap G$ equals $\mathfrak{B}_{g_{i}}$ and is a maximally compact Cartan subgroup of $G$. The first part of the theorem follows from the fact that any two maximally compact Cartan subgroups of $G$ are actually $G$-conjugate [13, Lemma 4.4]. We also remark for future use that maximally compact Cartan subgroups are necessarily connected.

Now for the second part. Assume that $G$ actually contains compact Cartan subgroups. Let $\sigma \in(B)$ be in any open double coset. Then there exists a Cartan subgroup $\mathfrak{S} \subseteq \mathbb{B S}$ so that $T=\mathfrak{S} \cap G=$ $\sigma^{-1} \mathfrak{B} \sigma \cap G$ is a compact Cartan subgroup of $G$. We appeal to the discussion in [13, pp. 1142ff]. First of all let $u_{1}, \cdots, u_{n} \in \mathbb{S}$ be a set of representatives for the complex Weyl group $W_{\leftrightarrow}=N_{\Theta}(\mathfrak{S}) / \mathfrak{S}$. Then the set $\left\{\mathfrak{B} \sigma u_{i} G\right\}_{i=1, \cdots, n}$ exhausts the collection of open double cosets, and $\mathfrak{B} \sigma u_{i} G=\mathfrak{B} \sigma u_{j} G \Leftrightarrow u_{i} u_{j}^{-1} \in N_{G}(T)$. In particular there are exactly $\#\left(W_{G}\right) / \#\left(W_{G}\right)$ open orbits. Furthermore, we have

$$
G \cap \sigma^{-1} \mathfrak{B} \sigma=G \cap u_{i}^{-1} \sigma^{-1} \mathfrak{B} \sigma u_{i}, i=1, \cdots, n .
$$

Indeed let $\sigma^{-1 \mathfrak{B} \sigma}=\mathfrak{S} \mathfrak{R}$ be the Levi decomposition of the Borel group $\sigma^{-1} \mathfrak{B} \sigma$. Then $G \cap \mathfrak{S} \mathfrak{R}=G \cap \mathfrak{F}=T$. Also

$$
G \cap u_{1}^{-1} \sigma^{-1} \mathfrak{B} \sigma u_{i}=G \cap u_{i}^{-1} \mathfrak{S} \mathfrak{N}\left(u_{i}=G \cap \mathfrak{S}\left(u_{i}^{-1} \mathfrak{R} u_{i}\right) .\right.
$$

Since the latter must be a maximally compact Cartan subgroup of $G$, it is necessarily equal to $G \cap \mathfrak{S}=T$. Thus we have 


$$
\begin{aligned}
\left.\pi(\chi)\right|_{G} & =\sum_{u_{i} \neq u_{j} \bmod W_{G}} \operatorname{Ind}_{\Re \sigma u_{i}}^{G} \chi^{\sigma u_{i}} \\
& =\sum_{s \in W_{W^{\prime} / W_{G}}} \operatorname{Ind}_{T}^{G} \chi^{\sigma s} .
\end{aligned}
$$

Our notation reflects the fact that $W_{\circlearrowleft}$ acts on $T$ as well as $\mathfrak{F}$.

Now we want to decompose the induced representations appearing on the right side of equations (1) and (2). We will achieve that by the method of Anh reciprocity-i.e., by analyzing the restrictions to $H$ of generic representations of $G$. Generic representations of $G$ are made up of various nondegenerate principal series and (perhaps) discrete series. The former are induced representations and Theorem 1.1 will again provide our main line of attack. The latter are not induced and require other methods for their analysis.

Before proceeding we make an observation needed in the sequel-namely $Z_{\mathfrak{B}} \cap G=Z_{G}$. This is because $g$ is a real form of $g$

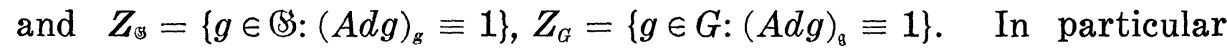
then

$$
\left.\chi^{\sigma s}\right|_{z_{G}}=\left.\chi^{\sigma}\right|_{z_{G}}=\left.\chi\right|_{z_{G}}
$$

for any $s \in W_{\Theta}, \sigma \in \mathbb{S}, \chi \in \hat{\mathfrak{B}}$.

3. Continuous spectrum. In this section we compute the continuous spectrum of $\left.\pi(\chi)\right|_{G}$ by restricting principal-series representations of $G$ to a maximally compact Cartan subgroup. These representations are given as follows. Let $P$ be a (proper) cuspidal parabolic subgroup of $G$ with Langlands decomposition $P=M A N$. For $\sigma \in \widehat{M}_{d}, \tau \in \hat{A}$ we form $\pi(\sigma, \tau)=\pi_{P}(\sigma, \tau)=\operatorname{Ind}_{P}^{G} \sigma \times \tau$, where $(\sigma \times$ $\tau)(\operatorname{man})=\tau(a) \sigma(m)$. The set $\left\{\pi_{P}(\sigma, \tau): \sigma \in \hat{M}_{d}, \tau \in \hat{A}\right\}$ constitutes the nondegenerate principal series representations of $G$ corresponding to $P$. There is some duplication in the set, and a "negligible proportion" may actually be reducible-but that need not concern us. Now we wish to analyze $\left.\pi(\sigma, \tau)\right|_{H}$, for $H$ a maximally compact Cartan subgroup. We must distinguish between the cases $H$ compact and $H$ noncompact. In the former our main result is

THEOREM 3.1. Let $T \leqq G$ be a compact Cartan subgroup. Let $P=M A N$ be a (proper) cuspidal parabolic subgroup of $G, \tau \in \hat{A}$, $\sigma \in \widehat{M}_{d}, \pi(\sigma, \tau)=\operatorname{Ind}_{P}^{G} \sigma \times \tau . \quad$ Then

$$
\left.\pi(\sigma, \tau)\right|_{T}=\left.\#(P \backslash G / T) \operatorname{Ind}_{Z_{G}}^{T} \sigma\right|_{Z_{G}} .
$$

We preface the proof with a lemma that will be useful on several occasions. 
Lemma 3.2. Let $P=M A N$ be a cuspidal parabolic subgroup of $G$. Let $K$ be a maximal compact subgroup of $G$ situated so that if $\mathfrak{g}=\mathfrak{l}+\mathfrak{q}$ is the corresponding Cartan decomposition and $\theta$ is the associated Cartan involution, then there is a $\theta$-invariant Cartan subgroup $C$ of $G$ inside $P$ with $C \cap \exp q=A$. Let $U \subseteq K$ be any compact connected abelian subgroup. Then there exists a principal orbit type for the (right) action of $U$ on $(K \cap M) \backslash K$ for which the principal stability subgroups are all $Z_{G}$.

Proof. By a fundamental result in compact Lie transformation groups (see e.g., [10]): the connected compact group $U$ acting differentiably on the compact connected manifold $(K \cap M) \backslash K$ must have a principal orbit type, say $O$. Since $U$ is abelian, all the stability groups $U_{x}, x \in \mathcal{O}$, are actually equal-say to $U_{1}$. By continuity, every element of $(K \cap M) \backslash K$ is pointwise fixed by every element of $U_{1}$. We must prove that $U_{1}=Z_{G}$. Clearly $Z_{G} \subseteq U_{1}$ (because $Z_{G} \subseteq$ $K \cap M)$. On the other hand, for each $u_{1} \in U_{1}$ we have $K \cap M \cdot u_{1}=$ $K \cap M$, i.e., $u_{1} \in K \cap M$. In particular $U_{1} \subseteq M$ and so it normalizes the subgroup $N$. Let $\bar{N}=\theta N$ be the opposed nilradical. Since $U_{1} \subseteq K, U_{1}$ also normalizes $\bar{N}$.

Now look at the canonical embedding

$$
\bar{N} \smile(K \cap M) \backslash K
$$

obtained by injecting $\bar{N}$ into $G$, then projecting onto $P \backslash G$ and finally using the identifications $P \backslash G=M A N \backslash K M A N \cong M \backslash K M \cong(K \cap M) \backslash K$. We observe that this embedding is equivariant for the action of $K \cap M$. Thus $U_{1}$ is a subgroup of $K \cap M$ that pointwise stabilizes $\bar{N}$. Applying $\theta$ we see that it fixes the elements of $N$ as well. The conclusion that $U_{1}=Z_{G}$ follows immediately then from Lemma 11 of [7].

Proof of Theorem 3.1. We choose a maximal compact subgroup $K$ of $G$ related to $P$ as in Lemma 3.2. Since all compact Cartan subgroups are mutually $G$-conjugate, it is no loss of generality to assume $T \subseteq K$. Now we apply Theorem 1.1 and Lemma 3.2. In fact $P \backslash G / T=(K \cap M) \backslash K / T$. Thus Lemma 3.2 shows that $P$ and $T$ are regularly related, and by Theorem 1.1 we have

$$
\begin{aligned}
\left.\pi(\sigma, \tau)\right|_{T} & =\left.\left(\operatorname{Ind}_{P}^{G} \sigma \times \tau\right)\right|_{T}=\int_{P \backslash G / T}^{\oplus} \operatorname{Ind}_{P_{u}}^{T}(\sigma \times \tau)^{u} \\
& =\left.\operatorname{dim} L_{2}(P \backslash G / T) \operatorname{Ind}_{Z_{G}}^{T}(\sigma \times \tau)\right|_{z_{G}} \\
& =\left.\sharp(P \backslash G / T) \operatorname{Ind}_{z_{G}}^{T} \sigma\right|_{z_{G}} .
\end{aligned}
$$

Next we pass to the situation in which the maximally compact 
Cartan subgroups are not compact. Let $H$ be one such. Recalling that $H$ is connected, we write $H=U S$ where $S$ is a maximal $\boldsymbol{R}$ split (nontrivial)(vector) subgroup and $U$ is the maximal compact (toral) subgroup of $H$. Let $P_{0}=M_{0} S N_{0}$ be a cuspidal parabolic subgroup of $G$ satisfying $P_{0} \supseteqq H=U S, M_{0} \supseteqq U$.

Proposition 3.3. If $\sigma \in \widehat{M}_{d}, \tau \in \hat{S}$ and $\pi(\sigma, \tau)=\operatorname{Ind}_{P_{0}}^{G} \sigma \times \tau$, then

$$
\left.\pi(\sigma, \tau)\right|_{I I}=\left.\#\left(P_{0} \mid G / H\right) \operatorname{Ind}_{Z_{G}}^{I I} \sigma\right|_{Z_{G}} \text {. }
$$

Proof. Consider the embedding

$$
\bar{N}_{0} \subset P_{0} \mid G
$$

given by injecting $\bar{N}_{0}$ into $G$, then projecting onto $P_{0} \mid G$. This map is $H$-equivariant and its range is open dense and co-null. Let $\Delta$ be the set of positive $\mathfrak{s}$-roots chosen so that $\overline{\mathfrak{n}}_{0}=\sum_{\alpha \in \Delta} \mathfrak{g}_{-\alpha}$. Every $X \in \overline{\mathfrak{n}}_{0}$ can be written uniquely $X=\sum_{\alpha \in \Delta} X_{-\alpha}, X_{-\alpha} \in \mathfrak{g}_{-\alpha}$. Put $\overline{\mathfrak{n}}_{0}^{\prime}=\left\{X \in \overline{\mathfrak{n}}_{0}: X_{-\alpha} \neq\right.$ $0 \forall \alpha \in \Delta\}$, an open dense co-null subset of $\overline{\mathfrak{n}}_{0}$. Now $a \cdot X=\sum e^{-\alpha(\log a)} X_{-\alpha}$, for $a \in S$. Thus if $X \in \overline{\mathfrak{n}}_{0}^{\prime}$, then $a \cdot X=X \Leftrightarrow \alpha(\log a)=0 \forall \alpha \in \Delta$. That can happen if and only if $a=1$.

Now the group $U$ preserves the root spaces $\mathfrak{g}_{-\alpha}$. On each $\mathfrak{g}_{-\alpha}$ we may choose a $U$-invariant norm function $\|\cdot\|_{\alpha}$. Next suppose that $u a \cdot X=X, u a \in H=U S, X \in \overline{\mathfrak{n}}_{0}^{\prime}$. Then $u a \cdot X_{-\alpha}=X_{-\alpha}, \forall \alpha \in A$, and

$$
\left\|X_{-\alpha}\right\|_{\alpha}=\left\|u a \cdot X_{-\alpha}\right\|_{\alpha}=\left\|a \cdot X_{-\alpha}\right\|_{\alpha}=e^{-\alpha(\log a)}\left\|X_{-\alpha}\right\|_{\alpha} .
$$

Therefore $a=1$ and $u \cdot X=X$. We apply Lemma 3.2 in order to assert the existence of a principal orbit type $\mathscr{O}$ for the action of $U$ on $\bar{N}_{0}$ with principal stability group $Z_{G}$. Clearly the set $\varnothing \cap$ $\exp \overline{\mathfrak{n}}_{0}^{\prime}$ is a principal orbit type for $H$ on which the principal stability groups are all $Z_{G}$. The proof is then completed by transferring $\bigcirc \cap \exp \overline{\mathfrak{n}}_{0}^{\prime}$ to $P_{0} \mid G$ via the embedding (7) and applying the Subgroup Theorem.

Now we generalize to an arbitrary nondegenerate principalseries representation.

THEOREM 3.4. Let $H=U S$ be a maximally compact (but not compact) Cartan subgroup of $G, P=M A N$ a cuspidal parabolic, $\sigma \in \widehat{M}_{d}, \tau \in \hat{A}$ and $\pi(\sigma, \tau)=\operatorname{Ind}_{P}^{G} \sigma \times \tau$. Then

$$
\left.\pi(\sigma, \tau)\right|_{H}=\left.\#(P \backslash G / H) \operatorname{Ind}_{Z_{G}}^{H} \sigma\right|_{z_{G}} .
$$

Proof. Let $P_{0}=M_{0} S N_{0}$ be a cuspidal parabolic corresponding to $H=U S$ as in Proposition 3.3. $P_{0}$ is a maximal cuspidal parabolic. 
By conjugating $P$ if necessary, we may assume that $S \subseteq A$. Furthermore-replacing $P$ by an associate parabolic if necessary-we may assume that the sets of positive a-roots and $\mathfrak{B}$-roots are compatible. That is we can find sets of positive a-roots, B-roots respectively, say $\Delta, \Delta_{0}$, such that

$$
\left.\alpha \in \Delta \cup\{0\} \Longrightarrow \alpha\right|_{\text {8 }} \in \Delta_{0} \cup\{0\} .
$$

Let $\mathfrak{p}, \mathfrak{p}_{0}$ be the Lie algebras of $P, P_{0}$. Then

$$
\begin{aligned}
\mathfrak{p} & =\sum_{\alpha \in \Delta \cup\{0\}} \mathfrak{g}_{\alpha} \\
\mathfrak{p}_{0} & =\sum_{\alpha \in \Delta_{0} \cup\{0\}} \mathfrak{g}_{\alpha} .
\end{aligned}
$$

Clearly $\mathfrak{p} \subseteq \mathfrak{p}_{0}$. But $M A=Z_{G}(A) \subseteq Z_{G}(S)=M_{0} S$. Also $N=\exp \mathfrak{n} \subseteq$ $\exp \mathfrak{p} \subseteq \exp \mathfrak{p}_{0} \subseteq P_{0}$. Hence $P \subseteq P_{0}$. This proves that once we fix a maximal cuspidal parabolic $P_{0}$, any other cuspidal parabolic is associate to a subgroup of $P_{0}$. But since associate parabolics determine equivalent collections of principal series representations, it is no loss of generality to assume $P \subseteq P_{0}$.

Now consider the canonical map

$$
P \backslash G \longrightarrow P_{0} \backslash G .
$$

$H$ acts on the right and this is obviously an $H$-equivariant map. But we saw in the proof of Proposition 3.3 that the space $P_{0} \mid G$ has a principal orbit type $\mathscr{Z}_{0}$ for the action of $H$ with principal stability group $Z_{G}$. Let $\mathscr{U}$ be its inverse image in $P \backslash G$. Then clearly the stability group for each $x \in \mathscr{U}$ is also $Z_{G}$. In particular the groups $P$ and $H$ are regularly related; and Theorem 1.1 yields

$$
\left.\pi(\sigma, \tau)\right|_{H}=\left.\left(\operatorname{Ind}_{P}^{G} \sigma \times \tau\right)\right|_{H}=\left.\sharp(P \backslash G / H) \operatorname{Ind}_{Z_{G}}^{I I} \sigma\right|_{Z_{G}} .
$$

This completes the proof.

Now we shall apply Anh reciprocity, together with Theorems 3.1 and 3.4 , to describe the continuous spectrum of $\left.\pi(\chi)\right|_{G}$. Let $P_{1}, \cdots, P_{r}$ denote a complete set of associativity classes of proper cuspidal parabolics.

THEOREM 3.5. Let $H$ be a maximally compact Cartan subgroup of $G$.

(i) Suppose $G$ has no discrete series ( $H$ noncompact). Then

$$
\left.\pi(\chi)\right|_{G}=\sum_{i=1}^{r} \int^{\oplus} n \#\left(P_{i} \backslash G / H\right) \delta(\sigma, \chi) \pi_{P_{i}}(\sigma, \tau) d \mu_{G, P_{i}}(\sigma, \tau)
$$


where $n$ is the number of open $\mathfrak{B}: G$ double cosets in $\mathbb{B}$,

$$
\delta(\sigma, \chi)= \begin{cases}\operatorname{dim} \sigma & \text { if }\left.\sigma\right|_{z_{G}}=\left.\chi\right|_{z_{G}} I \\ 0 & \text { if }\left.\sigma\right|_{z_{G}} \neq\left.\chi\right|_{z_{G}} I\end{cases}
$$

and $d \mu_{G, P_{i}}$ denotes the $P_{i}$-part of the Plancherel measure $\mu_{G}$ on $\hat{G}$.

(ii) Suppose $G$ has discrete series ( $H$ compact). Then

$$
\begin{gathered}
\left.\pi(\chi)\right|_{G}=\sum_{i=1}^{r} \int^{\oplus} \#\left(W_{\Theta} / W_{G}\right) \#\left(P_{i} \backslash G / H\right) \delta(\sigma, \chi) \pi_{P_{i}}(\sigma, \tau) d \mu_{G, P_{i}}(\sigma, \tau) \\
\bigoplus \sum_{\pi \in \hat{G}_{d}}^{\oplus} n(\pi, \chi) \pi
\end{gathered}
$$

where the multiplicities $n(\pi, \chi)$ of the discrete series are not yet specified.

Proof. If $G$ has a compact Cartan subgroup $T$, then for each $\pi \in \widehat{G}_{d}$, we can write

$$
\left.\pi\right|_{T}=\sum_{\chi \in \hat{T}}^{\oplus} n(\pi, \chi) \chi
$$

But equations (4), (8) and (11) show that for a generic set of irreducible representations $\pi \in \hat{G}$ we can expand

$$
\left.\pi\right|_{H}=\int_{\chi \in \hat{I I}}^{\oplus} n(\pi, \chi) \chi d \chi
$$

where $H$ is a maximally compact Cartan subgroup of $G$ and $d \chi$ is Haar measure on $\hat{H}$. The cardinals $n(\pi, \chi)$ are given explicitly for principal series $\pi$ by Theorems 3.1 and 3.4 ; but at this point they are not given for discrete series $\pi \in \widehat{G}_{d}$ (see however $\S \S 4,5$ ). Applying Anh reciprocity $[1, \S \mathrm{I}]$ we obtain:

$$
\operatorname{Ind}_{H I}^{G} \chi=\int_{\hat{G}}^{\oplus} n(\pi, \chi) \pi d \mu_{G}(\pi) \text { a.a. } \chi \in \hat{H} .
$$

Now if $H=T$ is compact, then "a.a. $\chi \in \hat{H}$ " means "all $\chi \in \hat{H}$." In fact equation (12) holds for all $\chi \in \hat{H}$ for noncompact $H=U S$ as well. This is because the representation $\operatorname{Ind}_{H}^{G} \chi$ is independent of $\left.\chi\right|_{S}$-which is the content of [7, Theorem 3]. The argument is finished by combining the preceding discussion with Theorem 2.1 and equation (3), and observing that the multiplicities (in the continuous spectrum) only depend (as shown in Theorems 3.1 and 3.4) on the central character.

To summarize we have determined the continuous spectrum of 
$\left.\pi(\chi)\right|_{G}$ for any $\chi \in \hat{\mathfrak{B}}$. We shall comment on the "size" of the multiplicities $n \#\left(P_{i} \mid G / H\right) \delta(\sigma, \chi)$ in $\S 5$. But first we need to investigate the discrete spectrum.

4. Discrete spectrum. We still wish to exploit Anh reciprocityso we need to compute $\left.\pi\right|_{T}$ for $\pi \in \widehat{G}_{d}, T$ a compact Cartan subgroup. That is we seek the $T$-types of discrete series representations and their multiplicities. The Subgroup Theorem is of no use here since discrete series representations are not induced. Of course one can write down an "answer"-namely, just combine the Blattner formula for $K$-types of discrete series with the Kostant formula for $T$-types of representations of $K$. The resulting multiplicity formula is totally unwieldy and provides no usable information. On the other hand, direct computation of the $T$-types in certain special cases strongly suggests two general conjectures. Before stating them, we recall Harish-Chandra's parametrization of the discrete series as well as some general facts about roots.

Let $T$ be a compact Cartan subgroup inside a maximal compact subgroup $K$ of $G, T \subseteq K \subseteq G$. Let $\mathrm{t}$ be the Lie algebra of $T, \mathrm{t}^{*}=$ $\operatorname{Hom}_{R}(\mathrm{t}, i \boldsymbol{R})$ and $\mathscr{L} \cong \mathrm{t}^{*}$ the lattice defined by $\mathscr{L}=\left\{\lambda \in \mathrm{t}^{*}: \lambda(x) \in\right.$ $2 \pi i \boldsymbol{Z}$ if $\left.\exp _{\sigma} x=1_{G}\right\}$. $\mathscr{L}$ is $W_{\Theta}$-invariant and identified to $\hat{T}$ by $\chi(\exp x)=e^{\chi(x)}$. Let $\Phi \leqq t^{*}=\operatorname{Hom}_{C}(\mathrm{t}, C)$ be the (connected) set of roots for ( $(\mathfrak{g}, \mathfrak{t})$. A root $\alpha \in \Phi$ is called compact (resp. noncompact) according as the corresponding root space $\boldsymbol{q}_{\alpha}$ lies in $\boldsymbol{t}$ (resp. $\boldsymbol{q}$ ). We write $\Phi^{c}\left(\right.$ resp. $\left.\Phi^{n}\right)$ for the set of compact (resp. noncompact) roots. Now suppose an order has been introduced in $t^{*}$ with respect to which $\Delta \subseteq \Phi$ is the set of positive roots. We put $\Delta_{n}=\Phi^{n} \cap \Delta, \Delta_{c}=$ $\Phi^{c} \cap \Delta$. If we write $\rho=1 / 2 \sum_{\alpha \in \Delta} \alpha$, then the set $\mathscr{L}+\rho$ is independent of the choice of ordering. We shall assume-for notational convenience only (this restriction plays no real role in the ensuing results)-that $\rho \in \mathscr{L}$. We also put $\rho_{n}=1 / 2 \sum_{\alpha \in \Delta_{n}} \alpha, \rho_{c}=1 / 2 \sum_{\alpha \in \Delta_{c}} \alpha$, so that $\rho=\rho_{n}+\rho_{c}$. Next we set $\mathscr{L}^{\prime}=\left\{\chi \in \mathscr{L}: s \chi \neq \chi \forall s \in W_{\Theta}, s \neq 1\right\}$. The set of regular elements $\mathscr{L}^{\prime}$ is also $W_{\circledast}$-invariant. Harish-Chandra has constructed (in [5]) a bijective correspondence $\lambda \rightarrow \pi_{\lambda}, \mathscr{L}^{\prime} / W_{G} \rightarrow$ $\hat{G}_{d}$.

Now when $G / K$ has a hermitian symmetric structure, there is a natural realization of certain of the discrete series representations $\pi_{\lambda}$ in spaces of holomorphic (or conjugate holomorphic) functions. These are enumerated as follows. When $G / K$ is hermitian symmetric, it is possible to choose the set of positive roots $\Delta$ so that

$$
\alpha, \beta \in \Delta_{n} \Longrightarrow \alpha+\beta \notin \Delta \text {. }
$$

The representations corresponding to the chambers 


$$
\begin{aligned}
& \left\{\lambda \in \mathscr{L}^{\prime}:\langle\lambda, \alpha\rangle<0 \quad \forall \alpha \in \Delta_{n}\right\} \\
& \left\{\lambda \in \mathscr{L}^{\prime}:\langle\lambda, \alpha\rangle>0 \quad \forall \alpha \in \Delta_{n}\right\}
\end{aligned}
$$

have been explicitly realized (originally by Harish-Chandra in [4]) in spaces of holomorphic (resp. conjugate holomorphic) functions. Moreover, modulo the action of $W_{G}$, there is exactly one chamber of each in $\mathscr{L}^{\prime}$. We refer to such representations $\pi_{\lambda}$ collectively as the holomorphic discrete series. We refer to the remaining discrete series-or all discrete series when $G / K$ is not hermitian symmetricas nonholomorphic discrete series.

Conjecture 4.1. (a) For every nonholomorphic discrete series representation $\pi \in \widehat{G}_{d}$, the restriction $\left.\pi\right|_{T}$ is quasi-equivalent to $\left.\operatorname{Ind}_{z_{G}}^{T} \pi\right|_{z_{G}}$.

(b) For every $\chi \in \hat{T}$, the set $\left\{\pi \in \hat{G}_{d}: \pi\right.$ is holomorphic and $\chi$ is a $T$-type of $\pi$ \} is finite.

In this section I shall give: (i) a proof of a slightly weaker result than (a) in a very special case; and (ii) a proof of (b) in complete generality that is due to Nolan Wallach. We start with the latter.

THEOREM 4.2. Let $\chi \in \hat{T}$. Then the set of holomorphic discrete series which have $\chi$ as a T-type is a finite set.

Proof. (Wallach.) We are in the case that $G / K$ is hermitian symmetric, $G$ simple. As before let $\Phi$ be the set of roots for $(\mathfrak{g}, \mathfrak{t})$. We may find a set $\Delta \leqq \Phi$ of positive roots (satisfying (13)) so that if $\alpha_{1}, \cdots, \alpha_{l}$ are the simple roots, then the largest root is of the form $\left(\sum_{i=1}^{l-1} n_{i} \alpha_{i}\right)+\alpha_{l}$. Moreover,

$$
\Delta_{n}=\left\{\sum_{i=1}^{l} m_{i} \alpha_{i} \in \Delta: m_{l}=1\right\} .
$$

Lemma 4.3. If $\alpha \in \Phi$, then $\alpha=\gamma_{1}-\gamma_{2}$ for some $\gamma_{1}, \gamma_{2} \in \Delta_{n}$.

Proof. For $\alpha \in \Phi_{n}$ this is obvious, so it is enough to show that any compact root may be written as the difference of two positive noncompact roots. Now we know that $[t, p] \subseteq p$ and $[p, p] \subseteq t$. Since $\boldsymbol{g}=[\boldsymbol{g}, \boldsymbol{g}]$, we must have $[\boldsymbol{p}, \boldsymbol{p}]=\boldsymbol{t}$ (see also $[6$, p. 207]). If we set

$$
\boldsymbol{p}^{+}=\sum_{\alpha \in-n} \boldsymbol{g}_{\alpha} \quad \boldsymbol{p}^{-}=\sum_{\alpha \in \epsilon_{-n}} \boldsymbol{g}_{-\alpha},
$$

then

$$
\left[\boldsymbol{p}^{+}, \boldsymbol{p}^{+}\right]=\left[\boldsymbol{p}^{-}, \boldsymbol{p}^{-}\right]=0 .
$$


Therefore $\left[\boldsymbol{p}^{+}, \boldsymbol{p}^{-}\right]=\boldsymbol{t}$ and the lemma follows.

LemmA 4.4. If $\alpha \in \Delta_{c}$, then $\left\langle\rho_{n}, \alpha\right\rangle=0$.

Proof. If $\beta \in \Delta_{n}$, then $\beta=\alpha_{l}+\sum_{i<l} m_{i} \alpha_{i}$. But if $j<l$, then

$$
s_{\alpha_{j}} \beta=s_{\alpha_{j}} \alpha_{l}+\sum_{i<l} p_{i} \alpha_{i}=\alpha_{l}+\sum_{i<l} q_{i} \alpha_{i} \in \Delta_{n}
$$

for suitable $p_{i}$ and $q_{i}$. Thus $s_{\alpha_{j}} \Delta_{n} \subseteq \Delta_{n}, j<l$. This implies $s_{\alpha_{j}} \rho_{n}=\rho_{n}$ and therefore that $\left\langle\rho_{n}, \alpha_{j}\right\rangle=0$. Since the simple roots $\alpha_{1}, \cdots, \alpha_{l-1}$ span $\Delta_{c}$, the lemma is proven.

Now let $q=\#\left(\Delta_{n}\right)$. Suppose that $Q \subseteq \Delta_{n}$ is a subset consisting of $q-1$ elements. Then $Q=\Delta_{n}-\{\gamma\}$ for some $\gamma \in \Delta_{n}$. For any subset $R \subseteq \Delta_{n}$, we set $\langle R\rangle=\sum_{\alpha \in R} \alpha$. Thus $\langle Q\rangle=2 \rho_{n}-\gamma$. Applying Lemma 4.4, we see that if $\alpha \in \Phi^{c}$, then

$$
\langle\alpha,\langle Q\rangle\rangle=-\langle\alpha, \gamma\rangle \text {. }
$$

Now let $S=\left\{\sum_{\beta \in \triangle_{n}} r_{\beta} \beta: r_{\beta} \in R, r_{\beta} \geqq 0\right\}$.

Lemma 4.5. There exist elements $\lambda_{1}, \cdots, \lambda_{l-1} \in S$ so that

$$
\left\langle\lambda_{i}, \alpha_{j}\right\rangle=\delta_{i j}, \quad 1 \leqq i, j \leqq l-1 .
$$

Proof. By Lemma 4.3, each $\alpha_{j}$ may be written $\alpha_{j}=\gamma_{j}-\gamma_{j}^{\prime}$ with $\gamma_{j}, \gamma_{j}^{\prime} \in \Delta_{n}$. Thus if $t_{j}=\gamma_{j}+\left\langle\Delta_{n}-\left\{\gamma_{j}^{\prime}\right\}\right\rangle$, then

$$
\left\langle t_{j}, \alpha_{i}\right\rangle=\left\langle\gamma_{j}, \alpha_{i}\right\rangle-\left\langle\gamma_{j}^{\prime}, \alpha_{i}\right\rangle=\left\langle\alpha_{j}, \alpha_{i}\right\rangle, \quad 1 \leqq i, j \leqq l-1 .
$$

Let $\mu_{1}, \cdots, \mu_{l-1}$ be the Gram-Schmidt orthonormalization of the $\alpha_{1}, \cdots, \alpha_{l-1}$. Then

$$
\mu_{i}=\alpha_{i}+\sum_{j<i} s_{i j} \alpha_{j}, \quad s_{i j} \in R
$$

where $s_{i j}>0$ because $\left\langle\alpha_{i}, \alpha_{j}\right\rangle<0$.

Now choose $\beta_{i}=\sum_{j=1}^{l-1} c_{j i} \alpha_{j} \in \sum_{1}^{l-1} R \alpha_{j}$ so that $\left\langle\beta_{i}, \alpha_{j}\right\rangle=\delta_{i j}$. We wish to show $c_{j i} \geqq 0$. But we can also write $\beta_{i}=\sum_{j=1}^{l-1} \boldsymbol{r}_{j i} \mu_{j}$ where

$$
r_{j i}=\left\langle\beta_{i}, \mu_{j}\right\rangle=\left\langle\beta_{i}, \alpha_{j}\right\rangle+\sum_{k<j} s_{j k}\left\langle\beta_{i}, \alpha_{k}\right\rangle \geqq 0 .
$$

Hence the equality

$$
\beta_{i}=\sum_{j=1}^{l-1} r_{j i}\left(\alpha_{j}+\sum_{k<j} s_{j k} \alpha_{k}\right)=\sum_{j=1}^{l-1} c_{j_{2}} \alpha_{j}
$$

guarantees that $c_{j i} \geqq 0$.

Set $\lambda_{i}=\sum_{j=1}^{l-1} c_{j i} t_{j} \in S$. Then 


$$
\begin{aligned}
\left\langle\lambda_{i}, \alpha_{j}\right\rangle & =\sum_{k} c_{k i}\left\langle t_{k}, \alpha_{j}\right\rangle=\sum_{k} c_{k i}\left\langle\alpha_{k}, \alpha_{j}\right\rangle \\
& =\left\langle\sum_{k} c_{k i} \alpha_{k}, \alpha_{j}\right\rangle=\left\langle\beta_{i}, \alpha_{j}\right\rangle \\
& =\delta_{i j} .
\end{aligned}
$$

Next we remark that for $\gamma, \alpha \in \Delta_{n}$, we have $\langle\gamma, \alpha\rangle \geqq 0$. Indeed if $\langle\gamma, \alpha\rangle<0$, then $\gamma+\alpha \in \Delta$. But then the coefficient of $\alpha_{l}$ in $\gamma+\alpha$ would be 2 .

Lemma 4.6. There exists $\sigma \in S$ so that $\left.\left\langle\sigma, \alpha_{i}\right\rangle\right\rangle 0$ for all $i=$ $1, \cdots, l$.

Proof. Select

$$
\sigma=\left(\sum_{i=1}^{l-1} \lambda_{i}\right)+\rho_{n}
$$

Then using Lemmas 4.4 and 4.5 we have $\left\langle\sigma, \alpha_{i}\right\rangle=1,1 \leqq i \leqq l-1$. In addition, by the above remark, we have $\left\langle\lambda_{i}, \alpha_{l}\right\rangle \geqq 0,1 \leqq i \leqq l-1$. Therefore

$$
\left\langle\sigma, \alpha_{l}\right\rangle=\sum_{1}^{l-1}\left\langle\lambda_{i}, \alpha_{l}\right\rangle+\left\langle\rho_{n}, \alpha_{l}\right\rangle \geqq\left\langle\rho_{n}, \alpha_{l}\right\rangle \geqq 0 .
$$

Now the equality $\left\langle\rho_{n}, \alpha_{l}\right\rangle=0$ cannot hold since $\left\langle\rho_{n}, \alpha_{i}\right\rangle=0,1 \leqq i \leqq$ $l-1$, and $\mathfrak{g}$ is not compact. Thus $\left\langle\rho_{n}, \alpha_{l}\right\rangle>0$.

Lemma 4.7. Let $C$ be a constant. Then the set

$$
Q=\left\{\beta=\sum_{i}^{l} n_{i} \alpha_{i}: n_{i} \in Z, n_{i} \geqq 0,\langle\beta, \alpha\rangle<C, \forall \alpha \in \Delta_{n}\right\}
$$

is a finite set.

Proof. Let $\beta \in Q, \beta=\sum_{i=1}^{l} n_{i} \alpha_{i}$; and let $\sigma$ be as in Lemma 4.6. Then $\sigma=\sum_{\alpha \in \Delta_{n}} m_{\alpha} \alpha, m \in \boldsymbol{R}, m_{\alpha} \geqq 0$. Now

$$
\langle\beta, \sigma\rangle=\sum_{1}^{l} n_{i}\left\langle\alpha_{i}, \sigma\right\rangle=\sum_{\alpha \in \Delta_{n}} m_{\alpha}\langle\beta, \alpha\rangle \text {. }
$$

We immediately conclude that $C$ is positive or there are no such $\beta$. Indeed

$$
\left\langle\beta, 2 \rho_{n}\right\rangle=\sum_{\alpha \in \Delta_{n}}\langle\beta, \alpha\rangle\langle q C
$$

and

$$
\left\langle\beta, 2 \rho_{n}\right\rangle=n_{l}\left\langle\alpha_{l}, 2 \rho_{n}\right\rangle
$$


Therefore

$$
q C>n_{l}\left\langle\alpha_{l}, 2 \rho_{n}\right\rangle \geqq 0 .
$$

Furthermore, we deduce that

$$
\langle\beta, \sigma\rangle \leqq \sum_{\alpha \in \Delta_{n}} m_{\alpha} C \equiv C_{1}, \quad C_{1}>0 .
$$

Hence $\sum n_{i}\left\langle\alpha_{i}, \sigma\right\rangle\left\langle C_{1}\right.$. But $\left\langle\alpha_{i}, \sigma\right\rangle>0$ all $i$; and thus for all $j$, $1 \leqq j \leqq l$

$$
n_{j}\left\langle C_{1} / D_{1}, \quad D_{1}=\min _{i}\left\langle\alpha_{i}, \sigma\right\rangle>0\right.
$$

Completion of Proof of Theorem 4.2. We write $s_{0}$ for the unique element of $W_{G}$ satisfying $s_{0} \Delta_{c}=-\Delta_{c}$. Now fix $\chi \in \hat{T}$. Let $\pi_{\lambda} \in \widehat{G}_{d}$ be a holomorphic discrete series representation. It is wellknown that $\lambda+\rho_{n}-\rho_{c}$ is the lowest $K$-type (i.e., lowest highest weight) for $\left.\pi_{\lambda}\right|_{K}$ and that $s_{0}\left(\lambda+\rho_{n}-\rho_{c}\right)$ is a lowest $T$-type. Therefore if $\chi$ occurs in $\left.\pi_{\lambda}\right|_{T}$, then necessarily

$$
\chi=s_{0}\left(\lambda+\rho_{n}-\rho_{c}\right)+\beta
$$

where $\beta$ is a sum of positive roots. But then $\langle\chi-\beta, \alpha\rangle>0, \forall \alpha \epsilon$ $\Delta_{n}$; that is

$$
\langle\beta, \alpha\rangle\left\langle C=\max _{r \in \Delta_{n}}\langle\chi, \gamma\rangle, \quad \forall \alpha \in \Delta_{n} .\right.
$$

By Lemma 4.7 there are only a finite number of $\beta$ that can satisfy such a condition. Hence the number of qualifying $\lambda$ is finite, and the theorem is completely proven.

Theorem 4.2 and Anh reciprocity say that $\operatorname{Ind}_{T}^{G} \chi$ contains only finitely many holomorphic discrete series. Applying Theorem 2.1 we see that in $\left.\pi(\chi)\right|_{G}$, the discrete spectrum contains only finitely many holomorphic discrete series. It may actually contain none--e.g., when $\chi \equiv 1$. A precise description of the exact set of holomorphic discrete series in the spectrum in complete generality does not seem feasible. The author has worked our several interesting cases-see e.g., $\mathrm{SU}(2,1)$ in $\S 6$.

We now turn to Conjecture 4.1(a). By reciprocity, an equivalent formulation is: for every $\chi \in \hat{T}$, the discrete spectrum of $\operatorname{Ind}_{T}^{G} \chi$ contains all nonholomorphic discrete series with central character $\left.\chi\right|_{z_{G}}$. The following result comes close to proving that, but a technical assumption severely limits its applicability (see Remark 1 following the proposition).

Proposition 4.8. Suppose that the maximal compact group $K$ 
is actually lsimple. Then the discrete spectrum of $\operatorname{Ind}_{T}^{G} \chi$ contains all discrete series of $G$ having central character $\chi_{Z_{G}}$, with at most finitely many exceptions.

Proof. First it is of course clear that whatever occurs in the (discrete) spectrum of $\operatorname{Ind}_{T}^{G} \chi$ must have central character $\chi_{z_{G}}$. Next consider the representation $\operatorname{Ind}_{T}^{K} \chi$. We claim that there is a finitedimensional representation $\tau$ of $K$ such that the representations

$$
\tau \oplus \operatorname{Ind}_{T}^{K} \chi \text { and }\left.\operatorname{Ind}_{Z_{K}}^{K} \chi\right|_{Z_{K}}
$$

are quasi-equivalent. Postponing the proof of that momentarily, we then conclude that the representations

$$
\operatorname{Ind}_{K}^{G} \tau \oplus \operatorname{Ind}_{T}^{G} \chi \text { and }\left.\operatorname{Ind}_{Z_{K}}^{G} \chi\right|_{Z_{K}}
$$

are quasi-equivalent. But it is a result of Harish-Chandra [5, Lemma 70] that $\operatorname{Ind}_{K}^{G} \tau$ can contain only finitely many discrete series in its spectrum. Furthermore the representations

$$
\left.\operatorname{Ind}_{Z_{K}}^{G} \chi\right|_{z_{K}} \text { and }\left.\operatorname{Ind}_{z_{G}}^{G} \chi\right|_{z_{G}}
$$

are also quasi-equivalent. This is because whenever $G / K$ is not hermitian symmetric (i.e., $K$ semisimple) it must be that $Z_{K} / Z_{G} \cong Z_{2}$, and then the quasi-equivalence $\left.\left.\operatorname{Ind}_{Z_{K}}^{G} \chi\right|_{Z_{K}} \approx \operatorname{Ind}_{Z_{G}}^{G} \chi\right|_{Z_{G}}$ can be demonstrated by a slight extension of the argument in [8, Theorem 2]. (One diagonalizes the adjoint action of $Z_{K} / Z_{G}$ on $q$ and employs the methods of [8, Prop. 4].) Thus we are reduced to substantiating the claim. Its proof will require the simplicity of $K$.

Let $\left\{\alpha_{1}, \alpha_{2}, \cdots, \alpha_{l}\right\}$ be a choice of positive compact simple roots of $(\mathfrak{g}, \mathfrak{t})$. Then for $\lambda, \mu \in \mathscr{L}$ we write $\lambda<\mu$ to mean $\mu-\lambda=$ $\sum_{i=1}^{l} n_{i} \alpha_{i}, n_{i} \in Z, n_{i} \geqq 0$. In particular $\left.e^{\mu}\right|_{Z_{K}}=\left.e^{\lambda}\right|_{Z_{K}}$. Put $\mathscr{L}^{+}=$the $K$-dominant (highest) weights $=\left\{\lambda \in \mathscr{L}: \lambda>s \lambda \forall s \in W_{G}\right\}=\{\lambda:\langle\lambda, \alpha\rangle \geqq$ $\left.0 \forall \alpha \in \Delta_{c}\right\} . \mathscr{L}^{+}$is a cross-section for $\mathscr{L} / W_{G}$ and parameterizes the space $\hat{K}$. Moreover for $\lambda \in \mathscr{L}^{+}$, the associated representation $\sigma_{\lambda} \in \hat{K}$ has the property that the spectrum of $\left.\sigma_{\lambda}\right|_{T}$ is

$$
\mathscr{W}_{K}(\lambda)=\left\{e^{\mu}: \mu \in \mathscr{L}, \lambda>s \mu \forall s \in W_{G}\right\} .
$$

Now fix $\mu \in \mathscr{L}$. Let $\mathscr{S}(\mu)=$ the spectrum of $\operatorname{Ind}_{T}^{K} e^{\mu}=\left\{\sigma_{\lambda}: \mu \in \mathscr{W}_{K}(\lambda)\right\}$. Put $\omega=\left.e^{\mu}\right|_{Z_{K}}$ and set

$$
\mathscr{S}_{\omega}=\left\{\sigma_{\lambda} \in \hat{K}:\left.\sigma_{\lambda}\right|_{Z_{K}}=\omega I\right\} .
$$

Clearly $\mathscr{S}(\mu) \subseteq \mathscr{S}_{\omega}$ and the burden of proof is to show that $\mathscr{S}_{\omega}$ $\mathscr{S}(\mu)$ is a finite set.

Let $\mathscr{L}_{r} \subseteq \mathscr{L}$ be the (compact root) lattice spanned by $\alpha_{1}, \cdots, \alpha_{l}$. Then $\left[\mathscr{L}: \mathscr{L}_{r}\right]<\infty$, and 


$$
\mu=\sum m_{i} \alpha_{i}, m_{i} \in \boldsymbol{Z} /\left[\mathscr{L}: \mathscr{L}_{r}\right]
$$

Moreover

$$
s \mu=\sum m_{i, s} \alpha_{i}, m_{i, s} \in Z /\left[\mathscr{L}: \mathscr{L}_{r}\right], \quad \forall s \in W_{G} .
$$

Now if $\sigma_{\lambda} \in \mathscr{S}_{\omega}$, then for any $s \in W_{G},\left.e^{\lambda}\right|_{z_{K}}=\left.e^{s \mu}\right|_{z_{K}}$, and so $\lambda-s \mu \epsilon$ $\mathscr{L}_{r}$. Thus if $\sigma_{\lambda} \in \mathscr{S}_{\omega}$ and

$$
\lambda=\sum n_{i}(\lambda) \alpha_{i}, n_{i} \in Z /\left[\mathscr{L}: \mathscr{L}_{r}\right],
$$

we have $n_{i}(\lambda)-m_{i, s} \in \boldsymbol{Z}$ for all $i, s$. Now the condition $\sigma_{\lambda} \notin \mathscr{S}(\mu)$ means that $\lambda>s \mu$ fails for some $s \in W_{G}$. That is, for some $i$ and some $s, n_{i}(\lambda)-m_{i, s}<0$. If we can show:

as $\lambda=\sum n_{i}(\lambda) \alpha_{i} \longrightarrow \infty$ in $\mathscr{L}^{+}$, then $n_{i}(\lambda) \longrightarrow+\infty$ for every $i$;

our proof would be done.

Let $\lambda_{1}, \cdots, \lambda_{l}$ be the set of fundamental highest weights for $K$. Then one knows that for each $i$

$$
\lambda_{i}=\sum_{k} q_{i k} \alpha_{k}, \quad q_{i k} \geqq 0 .
$$

Moreover because of our assumption that $K$ is simple we have $q_{i k}>0$ for all $i, k$. Therefore when we write

$$
\lambda=\sum m_{i}(\lambda) \lambda_{i}, \quad m_{i} \geqq 0
$$

we know that

$$
\begin{aligned}
\lambda & =\sum m_{i}(\lambda) \sum q_{i k} \alpha_{k} \\
& =\sum n_{i}(\lambda) \alpha_{i}
\end{aligned}
$$

where

$$
n_{i}(\lambda)=\sum_{j} m_{j}(\Lambda) q_{j i} \geqq\left(\min _{j} q_{j i}\right)\left(\sum_{j} m_{j}(\lambda)\right) .
$$

If $\lambda \rightarrow \infty$ in $\mathscr{L}^{+}$, then $\sum_{j} m_{j}(\lambda) \rightarrow+\infty$ and our result is at last completely proven.

REMARKs. 1. Unfortunately the assumptions $K$ simple and $\operatorname{rank} G=\operatorname{rank} K$ are satisfied simultaneously only when $G$ is locally isomorphic to one of the three exceptional groups $E V, E V I I I$ or FII. (The notation for exceptional groups is Helgason's [6].) Nevertheless the method of proof of Proposition 4.8 is very suggestive and I feel provides good evidence for the truth of conjecture 4.1(a).

2 . If $\chi \equiv 1$, then $K$ semisimple is enough to determine the spectrum of $\operatorname{Ind}_{T}^{G} \chi$. For then $Z_{K} / Z_{G} \cong Z_{2}$ is still valid and the quasiequivalence $\operatorname{Ind}_{T}^{G} 1 \approx \operatorname{Ind}_{Z_{K}}^{G} 1$ follows from [7, Cor. 7]. That the 
latter is quasi-equivalent to $\operatorname{Ind}_{z_{G}}^{G} 1$ we have already observed.

3. The explicit identification of irreducible subspaces of $L_{2}(\mathbb{S} / \mathfrak{B})$ on which $G$ acts by discrete series representations is of course an interesting problem. In [3] Cowling picks out some of them. Although he claims to have them all, it seems to me he has only realized holomorphic discrete series. But the regular representation of $(3)$ restricted to $G$ is equivalent to the regular irepresentation of $G$. Also the regular representation of $B S$ is the direct integral of the representations $\pi(\chi)$; and each $\left.\pi(\chi)\right|_{G}$ is a subrepresentation of the regular representation of $G$. It follows that every discrete series representation of $G$ must occur as an irreducible summand of $\left.\pi(\chi)\right|_{G}$ for some $\chi \in \hat{\mathfrak{B}}$. How indeed do we realize them?

5. Multiplicity. Here we shall explicitly compute the multiplicity of the continuous spectrum of $\left.\pi(\chi)\right|_{G}$, and make two comments on the multiplicity of the discrete spectrum.

THEOREM 5.1. The representation $\left.\pi(\chi)\right|_{G}$ has uniform continuous spectrum. It is of infinite multiplicity, except when $G$ is locally isomorphic to $\mathrm{SL}(2, \boldsymbol{R})$ or $\mathrm{SL}(2, \boldsymbol{C})$-in which cases the multiplicity is 2 or 1 respectively.

Proof. We refer back to Theorem 3.5. Our first observation is that $\operatorname{dim} \sigma=\infty$ for $\sigma \in \widehat{M}_{d}$ unless $P=M A N$ is a minimal parabolic subgroup. Thus the nonzero cardinals $\delta(\sigma, \chi)$ are infinite when $P$ is not minimal. Next we examine the cardinal $\#(P \backslash G / H)$ where $P$ is a minimal parabolic and $H$ is a maximally compact Cartan subgroup. When can it be finite? As usual we must consider the cases $H$ compact and $H$ noncompact separately.

Suppose first that $H=U S$ is not compact. Choose a maximal cuspidal parabolic $P_{0}=M_{0} S N_{0}$ as in Proposition 3.3. In the proof of that proposition we demonstrated that $\operatorname{dim} H \leqq \operatorname{dim} G / P_{0}$. Therefore the strict inequality $\operatorname{dim} H<\operatorname{dim} G / P$ can only fail if $P$ is conjugate to $P_{0}$. This occurs only when $G$ contains exactly one conjugacy class of Cartan subgroups.

Next suppose $H=T$ is compact. If there exists more than one conjugacy class of noncompact Cartan subgroups, then we can find a nonminimal cuspidal parabolic subgroup $P_{1} \neq G$. By Lemma 3.2, $\operatorname{dim} T \leqq \operatorname{dim} G / P_{1}$. Therefore $\operatorname{dim} T<\operatorname{dim} G / P$ and so $\sharp(P \backslash G / T)=\infty$. Hence in both cases we are reduced to the situation of only one conjugacy class of noncompact Cartan subgroups. We examine these on a case by case basis. They are:

(i) $G=a$ complex group viewed as real;

(ii) $G=\mathrm{SO}(n, 1)^{0}, n \geqq 2$; 


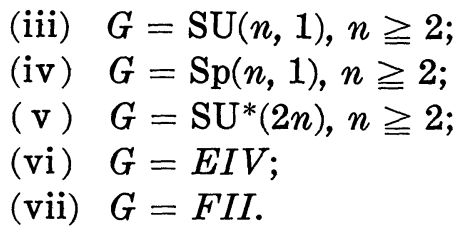

By explicit computation, we find that $\sharp(P \mid G / H)=\infty$ in all of these cases except when $G=S L(2, R)$ or $S L(2, C)$. Further computation in those cases reveals that: $\sharp(P \backslash G / H)=1$ in both instances; $\operatorname{dim} \sigma=1$; and the number of open $\mathfrak{B}: G$ double cosets is 2 and 1 in the real and complex cases respectively. That completes the proof of the theorem.

What about the multiplicity of the discrete spectrum? It is well-known that for holomorphic discrete series the multiplicity of $T$-types is finite. This is because (in the notation of $\S 4$ ) $p^{-}$acts trivially on the lowest highest weight space. Alternatively, one can use equation (15) to establish the finiteness. We shall see (by example in the next section) that although the holomorphic discrete series appears with finite multiplicity in $\left.\pi(\chi)\right|_{G}$, the multiplicity is not uniform. As for nonholomorphic discrete series, specific examples and the proof of Proposition 4.8 suggest that the multiplicity is uniform and always infinite. But at this point $I$ do not know how to prove that.

REMARK. In the case $\chi \equiv 1$ and $G / K$ not hermitian symmetric, the multiplicity can be determined. We leave it to the reader to augment Remark 2 of $\S 4$ so as to establish that $\left.\pi(1)\right|_{G}$ and $\operatorname{Ind}_{Z_{G}}^{G} 1$ are actually unitarily equivalent $(G \neq \mathrm{SL}(2, C))$.

6. An example. In this section we consider the specific case $G=\mathrm{SU}(2,1) \subseteq B S=\mathrm{SL}(3, C)$. We write out explicitly the spectrum and multiplicity for any restriction $\left.\pi(\chi)\right|_{G}$. This will illustrate all previous theorems and conjectures. Moreover in $\S 7$ we shall use these data to settle the main outstanding problem from [7], [8].

We set

$\mathbb{B S}=\mathrm{SL}(3, C)=$ the $3 \times 3$ complex matrices of determinant 1

$$
\begin{aligned}
& G=\mathrm{SU}(2,1)=\left\{g \in \mathbb{B}: g J^{t} \bar{g}=J, J=\left(\begin{array}{ccc}
1 & 0 & 0 \\
0 & 1 & 0 \\
0 & 0 & -1
\end{array}\right)\right\} \\
& K=S\left(U_{2} \times U_{1}\right)=\left\{\left(\begin{array}{ccc}
u_{11} & u_{12} & 0 \\
u_{21} & u_{22} & 0 \\
0 & 0 & c
\end{array}\right) \in G:\left(u_{i j}\right) \in U(2),|c|=1\right\}
\end{aligned}
$$




$$
\begin{aligned}
& \mathfrak{B}=\left\{\left(\begin{array}{lll}
a & x & z \\
0 & b & y \\
0 & 0 & c
\end{array}\right) \in \mathbb{B}\right\} \\
& \mathfrak{S}=\left\{\left(\begin{array}{lll}
a & 0 & 0 \\
0 & b & 0 \\
0 & 0 & c
\end{array}\right) \in \mathfrak{B}\right\} \\
& T=\left\{\left(\begin{array}{lll}
a & 0 & 0 \\
0 & b & 0 \\
0 & 0 & c
\end{array}\right) \in \mathfrak{S}:|a|=|b|=|c|=1\right\} \\
& Z_{G}=Z_{\circledast}=\left\{\left(\begin{array}{lll}
\zeta & \zeta & 0 \\
0 & \zeta & \zeta
\end{array}\right) \in T: \zeta^{3}=1\right\} .
\end{aligned}
$$

Given $\chi \in \hat{\mathfrak{B}}$ a unitary character, we define $\omega_{\chi}=\chi_{z_{G}}$ and $j(\chi)=$ the order of $\omega_{\chi}$. We have $N_{G}(T) / T \cong Z_{2}$ with representative

$$
s_{0}=\left(\begin{array}{rrr}
0 & 1 & 0 \\
-1 & 0 & 0 \\
0 & 0 & 1
\end{array}\right) \in K,
$$

and $N_{G}(\mathfrak{S}) / \mathfrak{S} \cong S_{3}$ with

$$
s_{1}=\left(\begin{array}{lll}
0 & 0 & 1 \\
1 & 0 & 0 \\
0 & 1 & 0
\end{array}\right)
$$

a generator of order 3. The double coset $\mathfrak{B} G$ is open in (B), e.g., because $\mathfrak{B} \cap G=T$. Therefore the set $\left\{s_{1}^{i}: i=0,1,2\right\}$ is a complete set of representatives for the open $\mathfrak{B}: G$ double cosets. According to Theorem 2.1

$$
\left.\pi(\chi)\right|_{G}=\sum_{i=0}^{2 \oplus} \operatorname{Ind}_{T}^{G} \chi_{s_{1}^{i}}^{i}
$$

Now we delineate the generic representations of $G$. There is only one conjugacy class of noncompact Cartan subgroups; and a corresponding minimal parabolic group is $P=M A N$ where

$$
\begin{aligned}
& M=\left\{\left(\begin{array}{lll}
\eta & 0 & 0 \\
0 & \eta^{-2} & 0 \\
0 & 0 & \eta
\end{array}\right) \in K:|\eta|=1\right\} \\
& A=\left\{\left(\begin{array}{lll}
e^{t} & 0 & 0 \\
0 & 1 & 0 \\
0 & 0 & e^{-t}
\end{array}\right): t \in R\right\}
\end{aligned}
$$




$$
N=\left\{\left(\begin{array}{ccc}
1 & \bar{z} & i u+\frac{|z|^{2}}{z} \\
0 & 1 & z \\
0 & 0 & 1
\end{array}\right): z \in \boldsymbol{C}, u \in \boldsymbol{R}\right\}
$$

Let $\sigma=\sigma_{n} \in \hat{M}$ and $\tau=\tau_{r} \in \hat{A}$ be described by

$$
\sigma\left(\begin{array}{lll}
\eta & 0 & 0 \\
0 & \eta^{-2} & 0 \\
0 & 0 & \eta
\end{array}\right)=\eta^{n}, n \in \boldsymbol{Z} \quad \tau\left(\begin{array}{lll}
e^{t} & 0 & 0 \\
0 & 1 & 0 \\
0 & 0 & e^{-t}
\end{array}\right)=e^{i t r}, r \in \boldsymbol{R} .
$$

Write $\pi(n, r)=\pi\left(\sigma_{n}, \tau_{r}\right)=\operatorname{Ind}_{P}^{G} \sigma_{n} \times \tau_{r}$. Then, since $\sharp(T \backslash G / P)=\infty$, Theorem 3.1 says that

$$
\left.\pi(n, r)\right|_{T}=\left.\infty \operatorname{Ind}_{z_{G}}^{T} \sigma_{n}\right|_{z_{G}} .
$$

According to Theorem 3.5, the continuous spectrum of $\left.\pi(\chi)\right|_{G}$ is exactly:

$$
\infty \int_{-\infty}^{\infty \oplus} \sum_{n \equiv j(x)}^{\oplus} \pi(n, r) d r
$$

Now we wish to describe the discrete spectrum for $\left.\pi(\chi)\right|_{G}$. We have seen that that is tantamount to describing the $T$-types of discrete series representations $\pi_{\lambda} \in \hat{G}_{d}$. The author has computed those by means of the Blattner formula (see e.g., [12]). Here are the results. First we have

$$
\mathrm{t}=\left\{\left(\begin{array}{ccc}
i u & 0 & 0 \\
0 & i v & 0 \\
0 & 0 & i y
\end{array}\right): u, v, y \in \boldsymbol{R}, u+v+y=0\right\} .
$$

In $t^{*}$ we chose coordinates according to the following convention:

$$
\begin{aligned}
\lambda_{m, n}\left(\begin{array}{ccc}
i u & 0 & 0 \\
0 & i v & 0 \\
0 & 0 & i y
\end{array}\right) & =m(i u-i v)+\sqrt{3} n(i u+i v) \\
& =(m+\sqrt{3} n) i u+(-m+\sqrt{3} n) i v, m, n \in \boldsymbol{R} .
\end{aligned}
$$

Then

$$
\begin{aligned}
\mathscr{L} & =\left\{\lambda_{m, n}: n \in \frac{\sqrt{3}}{6} \boldsymbol{Z}, m+\sqrt{3} n \in \boldsymbol{Z}\right\} \\
& =\boldsymbol{Z} \text {-span of } \lambda_{1,0} \text { and } \lambda_{-1 / 2, \sqrt{3} / 6} .
\end{aligned}
$$

We have chosen coordinates in this way so that the Killing form is (a multiple of) the usual Euclidean inner product. If we put $\alpha_{1}=\lambda_{1,0}, \alpha_{2}=\lambda_{-1 / 2, \sqrt{3} / 2}, \alpha_{3}=\lambda_{-1 / 2, \sqrt{3} / 2}$, then 


$$
\Phi=\left\{ \pm \alpha_{1}, \pm \alpha_{2}, \pm \alpha_{3}\right\} \quad \Phi^{c}=\left\{ \pm \alpha_{1}\right\} \quad \Phi^{n_{f}}=\left\{ \pm \alpha_{2}, \pm \alpha_{3}\right\}
$$

The lattice $\mathscr{L}_{r}$ is the $Z$-span of $\alpha_{1}, \alpha_{2}$, and

$$
\mathscr{L}_{r}=\left\{\lambda_{m, n}: n \in \frac{1}{2} \sqrt{3} Z, m-\frac{n}{\sqrt{3}} \in Z\right\}
$$

Writing $\lambda_{0}$ for $\lambda_{-1 / 2,1 \sqrt{3} / 6}$, i.e., the second basis vector of $\mathscr{L}$, we 'see that $\alpha_{2}=3 \lambda_{0}+\alpha_{1}$ and $\left[\mathscr{L}: \mathscr{L}_{r}\right]=3$. The picture is (Fig. 1):

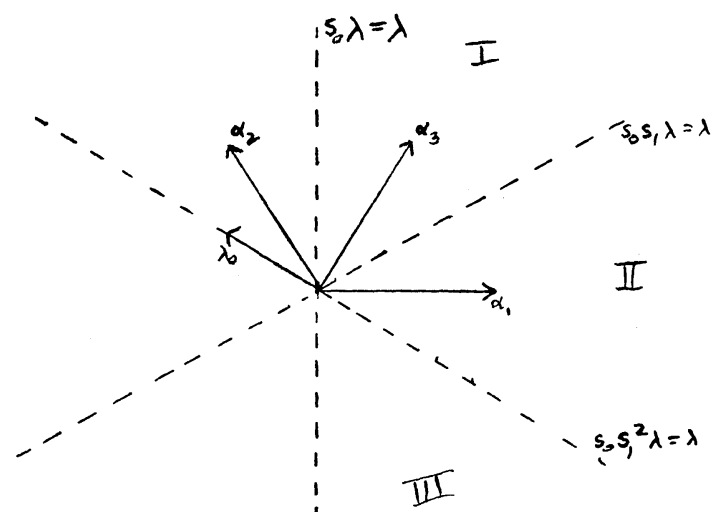

FIGURE 1

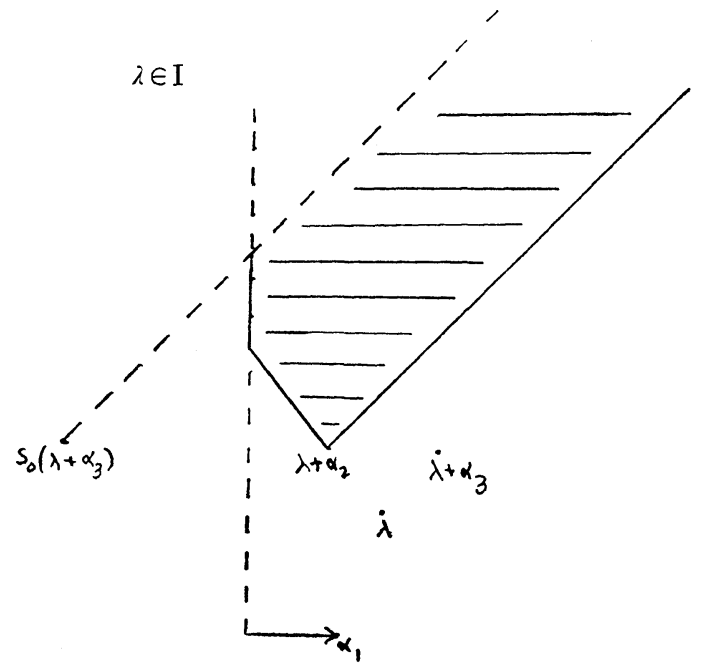

FIgure 2

We may take $\Delta=\left\{\alpha_{1}, \alpha_{2}, \alpha_{3}\right\}$ and the condition

$$
\alpha, \beta \in \Delta_{n} \Longrightarrow \alpha+\beta \notin \Phi
$$

is satisfied. One checks easily that $\mathscr{L}^{+}$is the closure of $I \cup I I \cup$ 


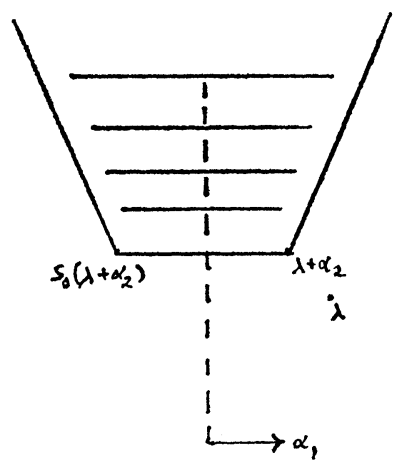

FiguRE 3

$I I I$; and that the chambers $I$ and $I I I$ give rise to holomorphic discrete series, chamber $I I$ to nonholomorphic discrete series. The shaded regions in the diagrams in Fig. 2 represent the set of $K$-types (i.e., the highest weights of the representations occurring in $\left.\pi_{\lambda}\right|_{K}$ ) for $\lambda=\lambda_{m, n} \in I$ or $\lambda=\lambda_{m, n} \in I I$. The corresponding diagram for $\lambda \in I I I$ is very similar to that of $I$. In all cases it is of course understood that $\mu \in \mathscr{L}$ in the shaded region is to be counted $\Leftrightarrow \mu \equiv$ $\lambda \bmod \mathscr{L}_{r}$. The $K$-multiplicities in all cases are 1 . In order to find the $T$-types, we only need take the $W_{G}$-convex hull of the shaded region. For the chamber $I I$ that gives all $\mu \in \mathscr{L}$ which are congruent to $\lambda \bmod \mathscr{L}_{r}$. For $\lambda \in I$, we get Fig. 3. Again $\mu \in \mathscr{L}$ is counted only when it is conjugate to $\lambda$ modulo $\mathscr{L}_{r}$.

The multiplicity of a $T$-type $\mu=\lambda_{m, n}$ is the number of $m_{1}$ 's satisfying: $\lambda_{m_{1}, n}$ is a $K$-type, $\lambda_{m_{1}, n} \equiv \mu \bmod \mathscr{L}_{r}$, and $|m| \leqq m_{1}$. Obviously that number is infinite for $\lambda \in I I$, but finite for $\lambda \in I$. In the latter case it's possible to write down specific multiplicities of the $T$-types, although the actual numbers are not so enlightening. The most important fact to note is this: although the multiplicities of the holomorphic discrete series occurring in $\left.\pi(\chi)\right|_{G}$ are finite, they are not uniform. For example, if $n>1$, then in $\left.\pi\left(n \alpha_{2}+n \alpha_{3}\right)\right|_{\mathrm{su}(2,1)}$, the representation $\pi_{j \alpha}, j=1,2, \cdots, n$, occurs with multiplicity $j$. Note finally that the number $\sup \{n$ : ᄏholomorphic discrete series $\pi$ occurring with multiplicity $n$ in $\left.\left.\pi(\chi)\right|_{G}\right\}$ is not a bounded function of $\chi$.

7. A counterexample. In two papers [7], [8] I considered the problem of decomposing the canonical unitary representation of a semisimple Lie group on $L_{2}$ of its Lie algebra. In the notation of this paper, the problem is to decompose the representation

$$
T_{G}(g) f(x)=f\left(A d g^{-1}(x)\right), g \in G, x \in \mathfrak{g}, f \in L_{2}(\mathfrak{g}) .
$$

The $L_{2}$ space is with respect to Lebesgue measure on $g$ (which is 
G-invariant). The working conjecture was that

$$
T_{G} \cong \operatorname{Ind}_{z_{G}}^{G} 1 \text {. }
$$

Indeed in the course of [7], [8] the conjecture was established for all the simple real Lie groups except $\mathrm{SU}(m, n), \mathrm{SO}^{*}(4 n+2)$ and EIII. However the conjecture is false in general. We shall use the $T$-type diagram constructed in $\S 6$ to demonstrate that.

Let $G=\mathrm{SU}(2,1)$ as in $\S 6$. According to [7, Thm. 9] we have

$$
T_{G} \cong \infty \operatorname{Ind}_{M}^{G} 1 \oplus \infty \operatorname{Ind}_{Z_{K}}^{G} 1 \text {. }
$$

We are going to produce a discrete series representation, with trivial central character, whose restriction to $M$ and also to $Z_{K}$ does not contain a fixed vector. In fact we shall look at holomorphic discrete series $\pi_{\lambda}$ arising from $\lambda$ in chamber $I$. No such representation has the zero vector as a $Z_{K}$-weight. We now compute for which $\lambda$, the restriction $\left.\pi_{\lambda}\right|_{M}$ contains a fixed vector.

First of all we may restrict attention to the lattice $\mathscr{L}_{r}$ since all central characters are trivial. Next we ask: for which $\chi \in \hat{T}$ is $\left.\chi\right|_{M}=1$. Recall that

$$
M=\left\{u_{\theta}=\left(\begin{array}{ccc}
e^{i \theta} & 0 & 0 \\
0 & e^{-2 i \theta} & 0 \\
0 & 0 & e^{i \theta}
\end{array}\right)\right\} ;
$$

so that if $\chi \leftrightarrow \lambda_{m, n}$, then $\chi\left(u_{\theta}\right)=e^{m(3 i \theta)} e^{\sqrt{3} n(-i \theta)}=e^{i \theta(3 m-\sqrt{3} n)}$. Thus $\left.\chi\right|_{M}=$ 1 if and only if $3 m-\sqrt{3} n=0$, or $n=\sqrt{3} m$. Now examining the diagram (Fig. 3) of T-types for a holomorphic discrete series $\pi_{\lambda}, \lambda \in I$, we see that in order for $\left.\pi_{\lambda}\right|_{M}$ to contain a fixed vector, it must that $\lambda+\alpha_{2}$ lies "to the right of $\alpha_{3}$ ". More precisely: $\left.\pi_{\lambda_{m, n}}\right|_{M}$ contains the trivial representation $\Leftrightarrow n+\sqrt{\overline{3}} \leqq \sqrt{3} m<3 n$, which is roughly half the chamber $I$. This proves that $T_{G} \not \operatorname{Ind}_{z_{G}}^{G} 1$, since a portion of the discrete series is missing from $T_{G}$.

\section{REFERENCES}

1. N. Anh, Restriction of the principal series of $S L(n, C)$ to some reductive subgroups, Pacific J. Math., 38 (1971), 295-313.

2. D. Basu and S. D. Majumdar, The master analytic function and the Lorentz group, J. Math. Phys., 17 (1976), 185-192.

3. M. Cowling, The discrete series, preprint.

4. Harish-Chandra, Representations of semisimple Lie groups V, Amer. J. Math., 78 (1956), 1-41.

5. - Discrete series for semisiple Lie groups II, Acta Math., 116 (1966), 1-111.

6. S. Helgason, Differential Geometry and Symmetric Spaces, Academic Press, N. Y., 1962. 
7. R. Lipsman, On the unitary representation of a semisimple Lie group given by the invariant integral on its Lie algebra, Advances in Math. Supp. Studies, 6 (1979), 143-158.

8. On the unitarized adjoint representation of a semisimple Lie group II, Canad. J. Math., 29 (1977), 1217-1222.

9. R. P. Martin, On the decomposition of tensor products of principal series representations for real-rank one semisimple groups, Trans. Amer. Math. Soc., 201 (1975), 177-211.

10. D. Montgomery, Orbits of highest dimension, Annals of Math. Studies, no. 46, Princeton U. Press, Princeton, 1960.

11. C. C. Moore, Restrictions of Unitary Representations to Subgroups and Ergodic Theory, Lecture Notes in Physics, vol. 6, Springer Verlag, N. Y., 1970.

12. W. Schmid, A proof of Blattner's conjecture, Invent. Math., 31 (1975), 129-154.

13. J. A. Wolf, The action of a real semisimple group on a complex flag manifold I, Bull. Amer. Math. Soc., 75 (1969), 1121-1237.

Received January 6, 1978. Research supported by the National Science Foundation (MCS 77-01264) and by the Institute for Physical Science and Technology.

College Park, MD. 20742 


\section{PACIFIC JOURNAL OF MATHEMATICS}

\section{EDITORS}

DONALD BABBITT (Managing Editor)

University of California

Los Angeles, CA 90024

HUGo RossI

University of Utah

Salt Lake City, UT 84112

C. C. MOORE and ANDREW OGG

University of California

Berkeley, CA 94720
J. DugundJI

Department of Mathematics

University of Southern California

Los Angeles, CA 90007

R. FinN and J. Milgram

Stanford University

Stanford, CA 94305

ASSOCIATE EDITORS
E. F. BECKENBACH
B. H. NeUmanN
F. WOLF
K. YoSHIDA

\section{SUPPORTING INSTITUTIONS}

UNIVERSITY OF BRITISH COLUMBIA

CALIFORNIA INSTITUTE OF TECHNOLOGY

UNIVERSITY OF CALIFORNIA

MONTANA STATE UNIVERSITY

UNIVERSITY OF NEVADA, RENO

NEW MEXICO STATE UNIVERSITY

OREGON STATE UNIVERSITY

UNIVERSITY OF OREGON
UNIVERSITY OF SOUTHERN CALIFORNIA

STANFORD UNIVERSITY

UNIVERSITY OF HAWAII

UNIVERSITY OF TOKYO

UNIVERSITY OF UTAH

WASHINGTON STATE UNIVERSITY

UNIVERSITY OF WASHINGTON

The Supporting Institutions listed above contribute to the cost of publication of this Journal, but they are not owners or publishers and have no responsibility for its content or policies.

Mathematical papers intended for publication in the Pacific Journal of Mathematics should be in typed form or offset-reproduced, (not dittoed), double spaced with large margins. Please do not use built up fractions in the text of the manuscript. However, you may use them in the displayed equations. Underline Greek letters in red, German in green, and script in blue. The first paragraph or two must be capable of being used separately as a synopsis of the entire paper. Please propose a heading for the odd numbered pages of less than 35 characters. Manuscripts, in triplicate, may be sent to any one of the editors. Please classify according to the scheme of Math. Reviews, Index to Vol. 39. Supply name and address of author to whom proofs should be sent. All other communications should be addressed to the managing editor, or Elaine Barth, University of California, Los Angeles, California, 90024.

50 reprints to each author are provided free for each article, only if page charges have been substantially paid. Additional copies may be obtained at cost in multiples of 50 .

The Pacific Journal of Mathematics is issued monthly as of January 1966. Regular subscription rate: $\$ 84.00$ a year (6 Vols., 12 issues). Special rato: $\$ 42.00$ a year to individual members of supporting institutions.

Subscriptions, orders for numbers issued in the last three calendar years, and changes of address shoud be sent to Pacific Journal of Mathematics, P.O. Box 969, Carmel Valley, CA 93924, U.S.A Old back numbers obtainable from Kraus Periodicals Co., Route 100, Millwood, NY 10546.

PUBLISHED BY PACIFIC JOURNAL OF MATHEMATICS, A NON-PROFIT CORPORATION

Printed at Kokusai Bunken Insatsusha (International Academic Printing Co., Ltd.). 8-8, 3-chome, Takadanobaba, Shinjuku-ku, Tokyo 160, Japan.

Copyright (C) 1980 by Pacific Jounal of Mathematics Manufactured and first issued in Japan 


\section{Pacific Journal of Mathematics \\ Vol. 89, No. $2 \quad$ June, 1980}

Frank Hayne Beatrous, Jr. and R. Michael Range, On holomorphic

approximation in weakly pseudoconvex domains................. 249

Lawrence Victor Berman, Quadratic forms and power series fields ...... 257

John Bligh Conway and Wacław Szymański, Singly generated antisymmetric operator algebras ....................... 269

Patrick C. Endicott and J. Wolfgang Smith, A homology spectral sequence for submersions . . . .................................

Sushil Jajodia, Homotopy classification of lens spaces for one-relator groups with torsion ................................ 301

Herbert Meyer Kamowitz, Compact endomorphisms of Banach

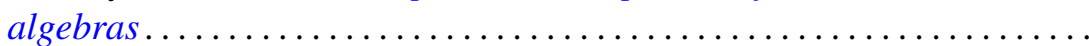

Keith Milo Kendig, Moiré phenomena in algebraic geometry: polynomial

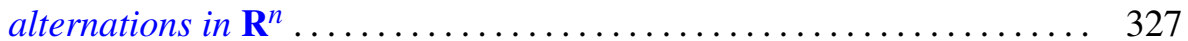

Cecelia Laurie, Invariant subspace lattices and compact operators....... 351

Ronald Leslie Lipsman, Restrictions of principal series to a real form . . . . . 367

Douglas C. McMahon and Louis Jack Nachman, An intrinsic

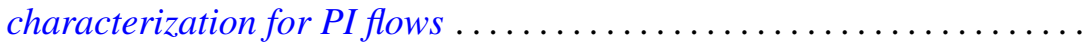

Norman R. Reilly, Modular sublattices of the lattice of varieties of inverse semigroups .................................... 405

Jeffrey Arthur Rosoff, Effective divisor classes and blowings-up of $\mathbf{P}^{2}$ 419

Zalman Rubinstein, Solution of the middle coefficient problem for certain

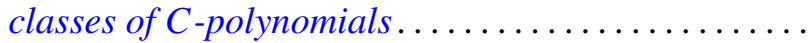

Alladi Sitaram, An analogue of the Wiener-Tauberian theorem for spherical transforms on semisimple Lie groups ................

Hal Leslie Smith, A note on disconjugacy for second order systems ...

J. Wolfgang Smith, Fiber homology and orientability of maps ...

Audrey Anne Terras, Integral formulas and integral tests for series of positive matrices. 